\title{
Correspondence
}

TO THE EDITOR, British Journal of Venereal Diseases

\section{Role of the VDRL test in the detection of} syphilis

Sir,

In the paper by $\mathrm{P}$ Diggory $(\mathrm{Br} J$ Vener Dis 1983;59:8-10) it was stated that, "the TPHA test results on sera from patients treated during the primary and early secondary stages of the disease usually become negative within one or two years." Work carried out in Edinburgh ${ }^{1}$ was misquoted in support of this statement. Our data showed that the TPHA test results did not become negative in any of the cases of treated syphilis. A more detailed study 2 confirmed these earlier findings: in none of 55 cases of early syphilis in which the TPHA result was positive before treatment did the test become negative after treatment.

We also have reservations regarding the general proposal that the VDRL test should be withdrawn from initial testing of syphilis except where early primary disease is suspected. Owing to the present low level of primary syphilis it could be argued that genitourinary medicine clinics themselves fall into this category: indeed this would appear to be the case at Southampton. Reports of the reactivity of the TPHA test in primary syphilis are conflicting. This may be due to variability in the IgM binding capacity of the TPHA reagents. It should also be stressed that the TPHA reaction, when it is positive in primary syphilis, is invariably only very weakly reactive whereas the VDRL result is usually unequivocally positive. Therefore screening with the TPHA test alone provides very little safety margin, and a slight reduction in test sensitivity could result in cases of early syphilis being missed. The limitations of relying solely on the TPHA test for screening would be much more apparent in a larger sample: "the theoretical risk of missing cases of primary syphilis at genitourinary clinics" could become a significant reality if such a policy were adopted nationally.

We maintain our earlier view 13 that particularly in clinics, where early detection is so important, the VDRL and TPHA tests are the best screens available. The comment that doctors in areas other than genitourinary medicine may be misled by a
VDRL-positive/TPHA-negative result into thinking that a patient has syphilis is not a valid criticism of the combined screening schedule: an explanatory comment added to the issued report should overcome any such misunderstanding.

Yours faithfully, H Young* D H H Robertson* $J$ M Huntert

*Departments of Bacteriology and Genitourinary Medicine, Edinburgh University, Edinburgh EH8 9AG

†Department of Genitourinary Medicine, Glasgow Royal Infirmary, Glasgow G31

\section{References}

1. Young $\mathrm{H}$, Henrichsen $\mathrm{C}$, Robertson $\mathrm{DHH}$ Treponema pallidum haemagglutination test as a screening procedure for the diagnosis of syphilis. Br J Vener Dis 1974; 50:341-6.

2. Hunter JM. The effect of treatment on the Treponema pallidum haemagglutination test in early syphilis. Scott med J 1979; 24: 307-12.

3. Robertson DHH McMillan A, Young $\mathrm{H}$ Henrichsen $C$. Clinical value of the Treponema pallidum haemagglutination test. $B r$ $J$ Vener Dis 1975; 51:79-82.

TO THE EDITOR, British Journal of Venereal Diseases

Continuing value of the VDRL test and biological false reactions

Sir,

Dr Diggory (this Journal, 1983;59:8-10) recommends dispensing with the Venereal Diseases Research Laboratory (VDRL) test in favour of the Treponema pallidum haemagglutination assay (TPHA) as, except in early primary syphilis, the VDRL test contributes little useful information and a positive reaction associated with a negative TPHA result may mislead many doctors into regarding their patients as having syphilis. May I suggest that this recommendation is much too sweeping. It takes no regard of the merits of two distinct serological reactions which, when both positive, contribute to a check that the correct sample has been examined and identified. Furthermore, the potential diagnostic value of a non-specific biological false positive reaction is discounted.
It must surely be rare for a microbiologis? to report a positive reaction without com menting on the probable clinical signifi cance. When the clinical information on the request form is inadequate the microbiologis $\$$ will generally discuss the relevance direcn with the clinician.

In a country where congenital syphilis i $\vec{\nabla}$ now exceedingly rare, a case can be made $\overrightarrow{\vec{e}}$ on the grounds of cost effectiveness fof limiting antenatal screening for treponemads disease to a single serological test. Although more specific than the VDRL test, theo TPHA is more subject to variation iris results between laboratories and to batchu variation in sensitivity. The VDRL test? though non-specific, is highly sensitive an\$ gives reproducible results even in relatively technically inexperienced hands. Perhaps io is time that serious consideration be giverf to discontinuing routine antenatal screening of all women and concentrating our efforts on a selected population of women ato greatest risk, for instance primiparae an $\$ 0$ unsupported women, screening not only at their first antenatal booking but again in the third trimester.

Unfortunately a biological false positiv? (BFP) reaction when found in pregnancy on elsewhere is often discounted as irrelevent and only rarely is the examination repeated 6 months or more later. As a chronic BFP reaction may, for example, be the first sero logical sign of impending connective tissue disease including rheumatoid arthritis, ip might be helpful to some of our patients if the significance of a BFP reaction weremore consistently pursued. Even an acute BFP reaction may herald underlying unsuspected pathology. This was dramatically demonstrated in Shrewsbure some months ago when a patient, who was believed to have been adequately treated for tabes dorsalis, was found on reattendance to have a significant rise in the VDRL titre. The temptation to repeat the course of antion treponemal antibiotic treatment was resisted. The patient was later shown to have had a "silent" coronary infarction t $\propto 0$ account for the non-specific rise it "reagin" antibodies.

Yours faithfully, C A Morris

Public Health Laboratory and Department of Microbiology, The Royal Shrewsbury Hospital, Shrewsbury, Shropshire SY3 $8 \mathrm{XH}$ 